\title{
Study on the Yoshida-Uemori Material Model of High-Tensile Steel Sheets by Shear Tests
}

\author{
Chih-Yi Chang ${ }^{1,}$, Ming-Hsiung $\mathrm{Ho}^{2}$ \\ ${ }^{1}$ Department of Mechanical Engineering, Nanya Institute of Technology, Taoyuan, Taiwan \\ ${ }^{2}$ Graduate School of Applied Technology, Nanya Institute of Technology, Taoyuan, Taiwan
}

\section{Email address:}

changci@nanya.edu.tw (Chih-Yi Chang), morrisho@nanya.edu.tw (Ming-Hsiung Ho)

*Corresponding author

\section{To cite this article:}

Chih-Yi Chang, Ming-Hsiung Ho. Study on the Yoshida-Uemori Material Model of High-Tensile Steel Sheets by Shear Tests. Science Discovery. Vol. 5, No. 1, 2017, pp. 59-68. doi: 10.11648/j.sd.20170501.20

Received: March 27, 2017; Accepted: April 17, 2017; Published: April 20, 2017

\begin{abstract}
In this research, the Yoshida-Uemori material models for four kinds of high-tensile steel sheet were analyzed by shear tests. The Yoshida-Uemori (Y-U) material model is currently the most important model in the simulation of stamp forming high-tensile steel sheets. It can comprehensively describe the Bauschinger effect and strain hardening behavior of metal during severe plastic deformation. Several commercial simulation software have built-in Y-U material models such as DYNAFORM and Pam-Stamp. The experimental curves must be obtained by the cyclic tension and compression test, and then the Y-U material parameters can be calculated by the regression operation. However, the parameters of the Y-U material model are difficult to obtain because the cyclic tension and compression test of thin sheet is prone to buckling phenomenon. This study first discuss the transform relation between shear test and tensile test, and then the Y-U material model is established by cyclic shear tests to avoid buckling. LS-DYNA numerical simulations of the shear tests were used to verify the accuracy of the material models. Four kinds of high-tensile steel sheet produced by China Steel Corporation are discussed, including JSC1180Y, JSC980Y, JAC590R and JSC440P, which cover a wide range of strength of the stamping plate. It further confirmed that the proposed research method does work.
\end{abstract}

Keywords: High-Tensile Steel Sheet, Yoshida-Uemori, Shear Test

\section{应用剪力试验于高张力钢板之Yoshida-Uemori材料性质研究}

\author{
張志毅 ${ }^{*}$, 何明雄 ${ }^{2}$ \\ 機械工程系, 南亞技術學院, 桃園市, 台灣 \\ 應用研究所, 南亞技術學院, 桃園市, 台灣 \\ 邮箱 \\ changci@nanya. edu. tw (張志毅), morrisho@nanya. edu. tw（何明雄）
}

摘要: 本文针对冲压产业常用的四种高张力钢板进行研究, 应用剪力试验探讨其Yoshida-Uemori材料性质, Yoshida-Uemori材料模型是目前高张力钢板冲压成形最重要的材料模型, 它能够完整地描述金属薄板在循环大塑性变 形下的包辛格效应 (Bauschinger effect), 几个常见的模拟软件例如DYNAFORM与Pam-Stamp已经内建Y-U材料模型, 但 因为 $\mathrm{Y}-\mathrm{U}$ 材料模型的参数必须藉由循环拉压试验取得实验曲线, 再经回归运算才能获得材料参数, 而且薄板的拉压试验 必须克服挫曲的问题, 有实际执行上的困难, 这使得Y-U材料模型参数不容易取得, 也造成许多冲压厂在模拟高张力钢 板成形制程时, 面临无材料系数可用的困境。本文首先探讨剪力试验与拉伸试验的转换关系, 再藉由往复式剪力试验 
建立Y-U材料模型, 以避免拉压试验的挫曲问题, 并透过LS-DYNA数值模拟剪力试验以验证其正确性。本文讨论的四种 高张力钢板分别为中钢公司所产制之JSC1180Y、JSC980Y、JAC590R与JSC440P, 广泛涵盖不同强度之冲压板材, 进一步 确认本文所提的研究方法确实可行。

关键词: 高张力钢板, Yoshida-Uemori, 剪力试验

\section{1. 引言}

2002年Yoshida与Uemori $[1,2]$ 针对循环大塑性变形 问题提出结合等向硬化与动态硬化的硬化准则, 建构出 Yoshida-Uemori材料模型, [1] 以软钢SPCC 与高强度钢 SPFC为例, 说明如何将五片薄板胶合后再配合特殊夹具进 行循环拉压试验, 并从实验曲线中计算可变弹性模数的参 数 $E a$ 与 $\xi$, 以及如何取得反向应力曲线并决定 $\mathrm{C}$ 值, [2]进 一步阐述 $\mathrm{Y}-\mathrm{U}$ 材料模型的理论背景, 并以明确的步骤与公 式, 说明如何取得 $\mathrm{Y}-\mathrm{U}$ 模型之各项参数, 此外, 也针对在 循环拉压试验曲线中, 初始降伏与再降伏曲线弯曲幅度差 异过大的情况提出修正模型, 分别以 $\mathrm{C} 1$ 与 $\mathrm{C} 2$ 控制这两部分 的曲线, 除此之外, 还针对降伏现象非常明显的传统钢材 加入一个新的参数 $r_{\text {initial }}$, 以便更精确模拟这类材料行为。 2003年Yoshida 与Uemori [3] 以不同硬化模型, 分别为等 向硬化、线性动态硬化, 等向硬化结合非线性动态硬化, 以及 $Y-U$ 动态硬化模型, 针对U形成形进行 CAE模拟, 获得 的回弹结果证实 $Y-U$ 模型确实比其他硬化模型更能模拟这 类具有明显包辛格效应与应变硬化的材料行为。

2008年 Shi et al. [4]发表在Numisheet 2008的文章, 针对 $\mathrm{Y}-\mathrm{U}$ 模型在拉伸过程中较大应变 $(\varepsilon>0.2)$ 的部分数值 模拟曲线会低于实验曲线的状况, 提出一组 $\mathrm{C} 1$ 与 $\mathrm{C} 2$ 参数以 修正Y-U模型, 而此C1与C2参数被LS-DYNA的MAT_125所采 用, 但此C1与C2 并非 [2] 所使用的C1与C2。2011年Hu et al. [5] 使用一套为LS-DYNA所设计的子程序 JSTAMP对汽车钣 件进行成形回弹CAE分析, 文中说明JSTAMP藉由大量实验 与模拟建立了一个 $\mathrm{Y}-\mathrm{U}$ 模型材料数据库, 但并未说明此数 据库中有多少种材料, 文中使用LS-SYNA内建的材料模型 MAT_125建立DP600材料的Y-U模型, 并利用实验与模拟证 实Y-U模型确实可比MAT_36与MAT_37更接近实验结果。

2010年Ghaei et al. [6, 7] 分别使用半隐式积分与全隐式 积分方法, 为ABAQUS撰写 $\mathrm{Y}-\mathrm{U}$ 模型的材料子程序UMAT (For ABAQUS Standard) 与VUMAT (For ABAQUS Explicit), 并以 NumiSheet 2005为例, 比较DP600使用等向硬化、等向硬 化结合非线性动态硬化, 以及Y-U动态硬化模型模拟的回 弹结果, 结果显示 $\mathrm{Y}-\mathrm{U}$ 模型模拟的回弹结果最接近实验结 果。2013年Chongthairungruang et al. [8] 考虑两种双相 钢JSC590R与JSC780Y以及一种软钢 JSC270C, 建立单轴拉 伸试验、液压突出试验 (Hydraulic bulge test)、圆盘压 缩试验与循环拉压试验, 取得MAT_122(Hi11 48), MAT_133(Barlat's Yield 2000) 以及 MAT_125(Y-U Kinematic) 三种材料模型参数, 并利用U形成形试验与 CAE 模拟比较回弹结果, 结论是 $\mathrm{Y}-\mathrm{U}$ 模型最接近实验的回弹结 果。
铝合金在汽车钣件上的应用范围正逐渐扩大, 但由于 弹性模数较低使得回弹非常明显, 而且容易发生应变局部 化的现象, 使得铝合金的成形性普遍较软钢差, 2011年 Tamura et al. [9] 将Y-U模型应用在5000与6000系列铝合 金, 从单轴向拉伸实验观察到铝合金AA6016-T4的异向性 系数 $r$ 与降伏应力具有强烈的平面异向性, 而A5052-0则相 较不明显, 循环拉压试验则可以观察到强烈的循环加工硬 化现象以及相对较不明显的包辛格效应。为了适应铝合金 这种材料性质, 作者选择Gotoh降伏函数配合 $\mathrm{Y}-\mathrm{U}$ 模型进行 循环拉压试验的CAE模拟, 并证实Y-U模型确实可准确模拟 这两种铝合金的材料行为, 唯文献中并未列出两种铝合金 的 $\mathrm{Y}-\mathrm{U}$ 模型材料参数值, 使得学者难以引用其结果作进一 步的应用与验证。从以上文献 [3-9] 可发现 Yoshida-Uemori模型在金属成形的CAE模拟具有重要地位, 因为 $\mathrm{Y}-\mathrm{U}$ 材料模型确实可以较精确反应高张力钢板的成形 回弹现象。

2009年Carbonnière et al. [10]针对Trip钢建立单轴 向拉伸试验、往复式剪力试验以及弯曲-反弯曲试验, 以 探讨弯曲一反弯曲以及纯剪试验中材料的加工硬化行为。 2012年Chang et al. [11]首先利用 [4] 中DP600的循环拉压 试验曲线, 依照 [2]所提供的方法计算 $\mathrm{Y}-\mathrm{U}$ 材料参数, 所得 Y-U参数虽然与 [4]不尽相同, 但应用在LS-DYNA模拟循环 拉压试验时, 仍然可以获得与实验曲线极为吻合的模拟曲 线, 可见对同一材料而言, $\mathrm{Y}-\mathrm{U}$ 参数并非仅有唯一正解。 此外, Chang et al. [11]提出比对实验曲线法, 利用 [10] 中Trip钢的实验曲线, 将往复式剪力试验转换成等效拉压 试验, 并利用转换后的等效拉压试验曲线回归计算 $\mathrm{Y}-\mathrm{U}$ 参 数, 再以此 $Y-U$ 参数模拟循环剪力试验, 获得跟实验曲线 非常一致的结果。2015年Chang et al. [12] 针对中钢公司 产制双相钢DP980建立单轴向拉伸试验、循环剪力试验与U 形成形试验, 以比对实验曲线法获得剪力试验与拉伸试验 的转换关系, 并利用转换后的等效拉压试验曲线回归计算 $\mathrm{Y}-\mathrm{U}$ 模型参数, 再以此 $\mathrm{Y}-\mathrm{U}$ 模型参数模拟剪力试验, 获得非 常一致的结果, 并且进一步从U形的成形模拟结果确认 $\mathrm{Y}-\mathrm{U}$ 模型参数的正确性。

然而仅仅二种钢材 (Trip与DP980) 的成果难以证实本 团队所提出的转换关系确实可用在其他钢材, 本文将针对 目前冲压业界常用高强度钢种进行试验与分析, 钢材将包 括中钢公司产制的低降伏比冷轧钢板JSC1180Y与JSC980Y, 高降伏比镀锌冷轧钢板JAC590R, 高深抽冷轧钢板JSC440P, 钢材的强度范围从 440 至 $1180 \mathrm{MPa}$, 将可进一步确认本研究 所提的转换关系确实可行, 而且利用剪力试验获取 $\mathrm{Y}-\mathrm{U}$ 模 型参数, 不仅可以避免拉压试验的挫曲问题, 较大的应变 量所获得的 $\mathrm{Y}-\mathrm{U}$ 模型参数, 才能确保应用在深抽拉制程模 拟的可行性。 


\section{2. 剪力试验与等效拉压试验之转换关系}

\section{1. von Mises降伏准则}

von Mises降伏准则一般应用在均质性及非异向性材 料, 其等效应力公式为:

$$
\bar{\sigma}=\sqrt{\frac{\left(\sigma_{1}-\sigma_{2}\right)^{2}+\left(\sigma_{2}-\sigma_{3}\right)^{2}+\left(\sigma_{3}-\sigma_{1}\right)^{2}}{2}}
$$

其中, $\sigma_{1}, \sigma_{2}, \sigma_{3}$ 为三个方向主应力, 等效应变公式为

$$
\begin{gathered}
\bar{\varepsilon}=\frac{\sqrt{2}}{3}\left[\left(\varepsilon_{x}-\varepsilon_{y}\right)^{2}+\left(\varepsilon_{y}-\varepsilon_{z}\right)^{2}+\left(\varepsilon_{z}-\varepsilon_{x}\right)^{2}\right. \\
\left.+\frac{3}{2}\left(\gamma_{x y}^{2}+\gamma_{y z}^{2}+\gamma_{z x}^{2}\right)\right]^{\frac{1}{2}}
\end{gathered}
$$

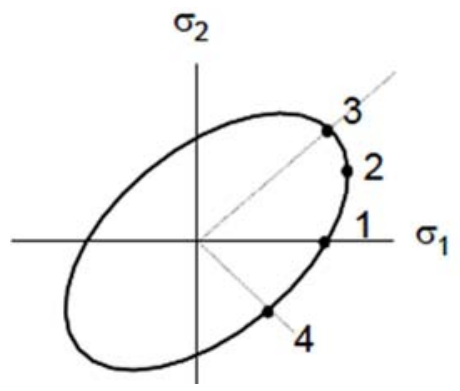

图1 von Mises降伏准则与不同的应力路径下所需之降伏应力。

图1为依照von Mises降伏准则, 主应力及次应力在不 同的应力路径下, 所需降伏应力值, 其中, 路径 1 为单轴 拉伸实验、路径 2 为平面应变拉伸试验、路径 3 为等双轴拉 伸测试、路径 4 为纯剪试验。

对于单轴拉伸而言, $\sigma_{2}=\sigma_{3}=0$ 所以 $\bar{\sigma}=\sigma_{1}$, 对于剪 力实验, $\sigma_{1}=-\sigma_{2}=\tau$, 带入方程式 (1), 得到

$$
\bar{\sigma}=\sqrt{3} \tau
$$

其中 $\sqrt{3}$ 就是纯剪负载情况下剪应力转换为等效应力 的转换系数, 将剪应变 $\gamma_{x y}=\gamma 、 \varepsilon_{x}=\varepsilon_{y}=\varepsilon_{z}=\gamma_{y z}=\gamma_{x z}=0$, 代入方程式 (2) 得到

$$
\bar{\varepsilon}=\frac{\gamma}{\sqrt{3}}
$$

上式代表纯剪负载情况下之剪应变乘以 $1 / \sqrt{3}$ 就可得 到等效应变, 可见透过von Mises降伏准则, 将剪力试验 的剪应力乘以 (转换系数), 剪应变乘以 (1/转换系数), 可 以转换成等效拉压试验的应力应变曲线。此外, 能量守恒 方程式为

$$
\bar{\sigma} d \bar{\varepsilon}=\sigma_{\alpha \beta} d \varepsilon_{\alpha \beta}
$$

对于剪力试验而言,

$$
\bar{\sigma} d \bar{\varepsilon}=2 \tau d \varepsilon_{x y}
$$

若将 (3) 式与 (4) 式代入 (5) 式, 可发现应力应变的转 换关系符合能量守恒定理。

\section{2. Hi1148降伏准则}

Hi1148降伏准则主要应用于软钢, 并考虑材料异向性, 其等效应力为

$\bar{\sigma}^{2}=(G+H) \sigma_{x x}^{2}-2 H \sigma_{x x} \sigma_{y y}+(F+H) \sigma_{y y}{ }^{2}+2 N \sigma_{x y}^{2}$

其中

$$
\left\{\begin{array}{l}
H=r_{0} G, \quad G=\frac{1}{r_{0}+1} \\
N=\frac{\left(r_{0}+r_{90}\right)\left(2 r_{45}+1\right)}{2 r_{90}\left(r_{0}+1\right)}, \quad F=\frac{H}{r_{90}}
\end{array}\right.
$$

对于剪力试验中, $\sigma_{x y}=\tau 、 \sigma_{x x}=\sigma_{y y}=0$ 代入方程式 (7) 得到 $\bar{\sigma}=\sqrt{2 N} \tau$, 即

$$
\bar{\sigma}=\sqrt{\frac{\left(r_{0}+r_{90}\right) \times\left(2 r_{45}+1\right)}{r_{90}\left(r_{0}+1\right)}} \tau
$$

且微等效应变为

$$
d \bar{\varepsilon}^{2}=\left\{\frac{\left[(F+H) d \varepsilon_{x x}^{2}+(H+G) d \varepsilon_{y y}^{2}+2 H d \varepsilon_{x x} \varepsilon_{y y}\right]}{F H+H G+G F}+\frac{2 d \varepsilon_{x y}^{2}}{N}\right\}
$$

对于剪力实验, $d \varepsilon_{x x}=d \varepsilon_{y y}=0$ 代入方程式 (10), 得

$$
\bar{\varepsilon}=\sqrt{\frac{2}{N}} \varepsilon_{x y}=2 \sqrt{\frac{r_{90}\left(r_{0}+1\right)}{\left(r_{0}+r_{90}\right)\left(2 r_{45}+1\right)}} \varepsilon_{x y}
$$

由式 (9) 与式 (11) 可以观察得知, 透过Hi11 48降伏准 则, 其转换系数为 $\sqrt{\left(r_{0}+r_{90}\right)\left(2 r_{45}+1\right) /\left[r_{90}\left(r_{0}+1\right)\right]}$, 而且 同样满足能量守恒定理。

\subsection{Barlat 89降伏准则}

Barlat 89降伏准则主要应用于如铝材这类低异向性 系数的材料, 其等效应力公式为

$$
2 \bar{\sigma}^{m}=a\left|K_{1}+K_{2}\right|^{m}+a\left|K_{1}-K_{2}\right|^{m}+c\left|2 K_{2}\right|^{m}
$$

其中

$$
\left\{\begin{array}{l}
K_{1}=\frac{\left(\sigma_{x x}+h \sigma_{y y}\right)}{2} \\
K_{2}=\sqrt{\left(\frac{\sigma_{x x}-h \sigma_{y y}}{2}\right)^{2}+\left(p \sigma_{x y}\right)^{2}}
\end{array}\right.
$$


对于剪力试验 $\sigma_{x y}=\tau$ 且 $\sigma_{x x}=\sigma_{y y}=0$, 代入上式得到 $K_{1}=0, K_{2}=p \tau$, 因此等效应力为

$$
\bar{\sigma}=(c / 2)^{1 / m}(2 p \tau)
$$

为了满足能量守恒定理, 将方程式 (14) 代入方程式 (6) 得到等效应变转换公式

$$
\bar{\varepsilon}=\frac{1}{p(c / 2)^{1 / m}} \varepsilon_{x y}
$$

其中,

$$
\left\{\begin{array}{l}
a=2-c, \quad c=\sqrt{\frac{r_{0}}{1+r_{0}} \frac{r_{90}}{1+r_{90}}} \\
h=\sqrt{\frac{r_{0}}{1+r_{0}} \frac{1+r_{90}}{r_{90}}}, \quad p=\frac{\sigma_{y}}{\tau_{s 1}}\left(\frac{2}{2 a+2^{m} c}\right)^{1 / m}
\end{array}\right.
$$

且 $\sigma_{y}$ 为单轴拉伸实验中材料沿辊压方向之降伏强度, $\tau_{s 1}$ 为剪力实验中材料沿辊压方向之剪力降伏强度, 对于 体心立方 (BCC) 钢种而言 $m=6$, 对于面心立方 (FCC)钢种 而言 $m=8$ 。

\section{4. Hi11 90降伏准则}

Hi1190降伏准则主要应用于低异向性系数材料如高 强度钢, 其等效应力公式为:

$$
\begin{aligned}
& \left(2 \sigma_{f}\right)^{m}=\left|\sigma_{1}+\sigma_{2}\right|^{m}+\left(1+2 r_{45}\right)\left|\sigma_{1}-\sigma_{2}\right|^{m} \\
& +\left|\sigma_{1}^{2}+\sigma_{2}^{2}\right|^{(m / 2)-1}\left[-2 a\left(\sigma_{1}^{2}-\sigma_{2}^{2}\right)+b\left(\sigma_{1}-\sigma_{2}\right) \cos 2 \alpha\right] \cos 2 \alpha
\end{aligned}
$$

或

$$
\begin{aligned}
& \left(2 \sigma_{f}\right)^{m}=\left|\sigma_{x x}+\sigma_{y y}\right|^{m}+\left(1+2 r_{45}\right)\left|\left(\sigma_{x x}-\sigma_{y y}\right)^{2}+4 \sigma_{x y}^{2}\right|^{m / 2} \\
& +\left|\sigma_{x x}{ }^{2}+\sigma_{y y}{ }^{2}+2 \sigma_{x y}^{2}\right|^{(m / 2)-1}\left[-2 a\left(\sigma_{x x}^{2}-\sigma_{y y}^{2}\right)+b\left(\sigma_{x x}-\sigma_{y y}\right)^{2}\right]
\end{aligned}
$$

其中,

$$
\left\{\begin{array}{l}
a=\frac{\left(2 \sigma_{f} / \sigma_{90}\right)^{m}-\left(2 \sigma_{f} / \sigma_{0}\right)^{m}}{4} \\
b=\frac{\left(2 \sigma_{f} / \sigma_{90}\right)^{m}+\left(2 \sigma_{f} / \sigma_{0}\right)^{m}}{4}-\left(2 \sigma_{f} / \sigma_{45}\right)^{m}
\end{array}\right.
$$

且 $\sigma_{f}$ 为等双轴拉伸降伏强度, $\sigma_{0}$ 为与辊压方向之单 轴拉伸降伏强度, $\sigma_{45}$ 为与辊压方向夹 45 度试片之单轴拉 伸降伏强度, $\sigma_{90}$ 为与辊压方向夹 90 度试片之单轴拉伸降 伏强度。若考虑剪力实验时, $\sigma_{x x}=\sigma_{y y}=0$ 代入方程式 (18) 得到等效应力公式

$$
\bar{\sigma}=\sigma_{f}=\sqrt[m]{\left(1+2 r_{45}\right)} \tau
$$

根据能量守恒方程式 (6), 可得到等效拉伸应变

$$
\bar{\varepsilon}=\frac{2}{\sqrt[m]{\left(1+2 r_{45}\right)}} \varepsilon_{x y}
$$

其中, $m=\ln \left[\left(1+2 r_{45}\right) /\left(\sigma_{f} / \tau_{s 1}\right)\right]$ 。

根据上述各种降伏准则等效应力与应变的推导, 各降 伏准则所需实验方法及量测数据整理如表 1 , 其中von Mises降伏准则不需任何量测实验及数据, Hi1148降伏准 则仅需单轴拉伸实验来量测辊压方向 $0 、 45$ 及 90 度的异向 性系数, Barlat 89降伏准则不仅需单轴拉伸实验, 还需 剪力实验来量测剪力降伏强度, Hil1 90 降伏准则除了上 述实验数据, 还要双轴拉伸实验来量测等拉双轴降伏强度, 此种实验设备精密且昂贵。

表 1 等效应力应变转换方法所需量测数据与实验方法。

\begin{tabular}{lll}
\hline 降伏准则 & 需要的数据 & 实验方法 \\
\hline von Mises & 无 & 无 \\
Hi11 48 & $r_{0}, r_{45}, r_{90}$ & 单轴拉伸试验 \\
Barlat 89 & $r_{0}, r_{45}, r_{90}$, 剪力降伏强度, 辊压方向单轴拉伸降伏强度。 & 单轴拉伸试验、剪力试验。 \\
\multirow{2}{*}{ Hi11 90} & $r_{0}, r_{45}, r_{90}$, 剪力降伏强度, 辊压方向单轴拉伸降伏强度, 辊压方向45度单轴拉伸降伏强度, & 等双轴拉伸试验、剪力试验、单轴 \\
& 辊压方向 90 度单轴拉伸降伏强度, 等拉双轴降伏强度 & 拉伸试验。 \\
\hline
\end{tabular}

\section{5. 比对实验曲线法}

[11]以Trip钢为例, 分别利用von Mises、Hill 48 与 Barlat' 98降伏准则所获得之转换系数, 将剪力试验转换 成等效拉伸试验, 但转换后的等效拉伸试验曲线与真实拉 伸试验曲线仍有明显的差距, 因而提出比对实验曲线法以 获得转换系数, 结果显示, 对Trip钢 [11]而言, 透过比对 剪力试验与拉伸试验实验曲线, 转换系数 1.8 所得到的等 效拉伸试验曲线与单轴拉伸实验曲线最接近。然而利用比
对实验曲线法所获得之转换系数会因材料不同而不同, 以 中钢公司所生产之DP980 [12] 为例, 转换系数为 $F=1.81$, 经转换之后的等效拉伸试验曲线与真实拉伸试验的曲线 极为吻合。可见剪力试验可透过 (22) 式的转换关系转成等 效拉伸试验, 唯其转换系数 $F$ 必须透过实验曲线比对而得。

$$
\left\{\begin{array}{l}
\bar{\sigma}=F \tau \\
\bar{\varepsilon}=\frac{\gamma}{F}
\end{array}\right.
$$




\section{3. 实验方法}

\section{1 . 单轴向拉伸实验}

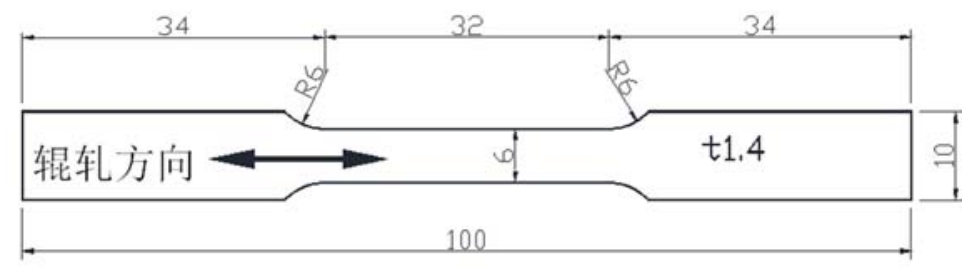

图2 单轴向拉伸试验的试件尺寸图。

本研究使用单轴向拉伸试验的试件尺寸如图2所示, 使用万能拉伸试验机型号为MTS 810 , 最大输出拉力为 $100 \mathrm{kN}$, 单轴向拉伸试验获得每种材料之拉伸应力应变曲 线, 是与单向剪力试验比对以获得转换系数的重要依据。 除此之外, 我们也准备了辊轧方向与拉伸方向成0度、45 度与 90 度的试片, 以拉伸试验测量薄板三种辊轧角度的异 向性系数 (anisotropic coefficient) $r_{0}, r_{45}, r_{90}$, 异向 性系数的定义如 (23) 式

$$
r=\frac{\varepsilon_{w}}{\varepsilon_{t}}
$$

假设材料体积不可压缩, 则三方向的应变和为 0 如 (9) 式所示

$$
\varepsilon_{l}+\varepsilon_{w}+\varepsilon_{t}=0
$$

其中 $\varepsilon_{l}, \varepsilon_{w}, \varepsilon_{t}$ 分别为长度、宽度与厚度方向的应变, 对薄板而言, 由于厚度太小, 以致于厚度方向的应变测量
误差较大, 因此可将异向性系数改写为 (10) 式, 可降低量 测的误差, 仅需使用准确度 $0.01 \mathrm{~mm}$ 的游标卡尺即可正确测 量。

$$
r=\frac{-\varepsilon_{w}}{\varepsilon_{l}+\varepsilon_{w}}
$$

单轴向拉伸试验所获得之材料性质表如表2所示。

\section{2. 往复式剪力试验}

本研究使用的剪力试验试片尺寸形状如图3所示, 是 属于双剪的夹持方式, 使用的夹具如图4所示, 由于高强 度钢的强度远高于一般软钢, 无法使用一般夹头, 必须特 别设计油压夹头才能避免试片滑脱, 庞大的夹具不仅拆装 困难, 更增加光学量测的困难, 最后仅能使用传统应变规

\begin{tabular}{|c|c|c|c|c|c|c|c|c|c|c|}
\hline 钢材 & $\begin{array}{l}\text { 弹 性 模 数 } \\
\text { (Mpa) }\end{array}$ & $\begin{array}{l}\text { 朴松比 } \\
\text { (假设值) }\end{array}$ & $\begin{array}{l}\text { 降伏强度 } \\
\text { (Mpa) }\end{array}$ & $\begin{array}{l}\text { 抗 拉 强度 } \\
(\mathrm{Mpa})\end{array}$ & $\begin{array}{l}\text { 最大 伸 } \\
\text { 长量 }\end{array}$ & $\begin{array}{l}\text { 测量 } r \text { 值之轴 } \\
\text { 向应变 }\end{array}$ & $r_{0}$ & $r_{45}$ & $r_{90}$ & $\begin{array}{l}\text { 平均异 向性 } \\
\text { 系数 } \mathrm{R}\end{array}$ \\
\hline JSC1180Y & 198166 & 0.3 & 960 & 1278 & $12.0 \%$ & $3 \%$ & 0.406 & 0.629 & 0.382 & 0.512 \\
\hline JSC980Y & 201652 & 0.3 & 870 & 1165 & 14. $0 \%$ & $8 \%$ & 0.295 & 0.729 & 0.789 & 0.635 \\
\hline JAC590R & 208433 & 0.3 & 430 & 740 & $26 \%$ & $8 \%$ & 0.500 & 0.201 & 0.456 & 0.339 \\
\hline JSC440P & 204923 & 0.3 & 290 & 577 & $32 \%$ & $8 \%$ & 1. 007 & 1.091 & 0.920 & 1. 027 \\
\hline
\end{tabular}
量测剪应变。

表2 单轴向拉伸试验材料性质表。

剪力试片中的凹槽是参考 [13]中的建议, 可避免剪力变形时的边缘效应, 图中H代表变形区的高度, H愈大代表变 形的截面积 (厚度 $\times \mathrm{H}$ ) 愈大, 对万能试验机的负载就愈大, 而且油压夹头必须施加更大力量才能避免试片滑脱, 但H愈 大，边缘效应对剪力变形中央的量测区影响愈小。

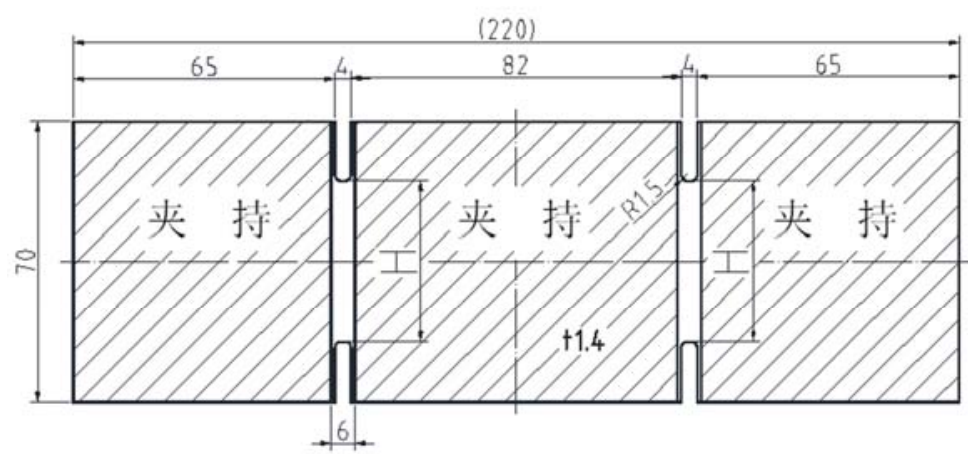

图3 剪力试验试片形状尺寸图。 


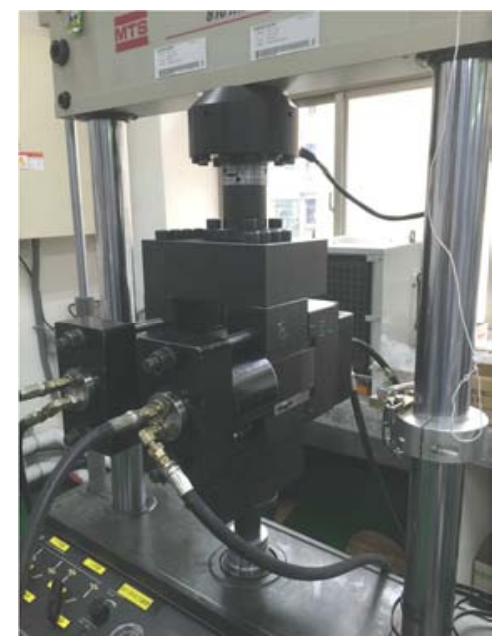

图4 剪力试验夹具图。
为了了解H对边缘效应的影响以取得合适的H值, 分别 使用不同试件 $\mathrm{H}=30,40,50,60 \mathrm{~mm}$ ，进行剪力试验有限元素 模拟, 并将变形区垂直中线与水平中线的剪应变与剪应力 绘图如图5与 6 。

剪力试验的应变测量范围主要在试件中央均匀变形 的区域, 此区域的应变变化愈小, 代表受边缘效应影响与 小, 从图5观察得知, $H$ 愈大, 延垂直中线的剪应变平直区 范围愈大。从图6观察得知, 沿着水平中线的剪应变分布 曲线几乎不随 $\mathrm{H}$ 值而改变, 而且接近平直的范围大约有 $2 \mathrm{~mm}$ 左右。可见H愈大, 中央区域受边缘效应的影响愈小, 但 对拉伸试验机而言, 变形截面积愈大, 拉伸试验机所需拉 力愈大, 因此两者必须取得平衡点, 图5的曲线显示, $\mathrm{H}=30 \mathrm{~mm}$ 剪应变曲线平直区大约 $10 \mathrm{~mm}$ 左右, $\mathrm{H}=40 \mathrm{~mm}$ 剪应变曲 线平直区大约 $15 \mathrm{~mm}$ 左右, 因此 $\mathrm{H}=40 \mathrm{~mm}$ 应该是比较合适的选 择。

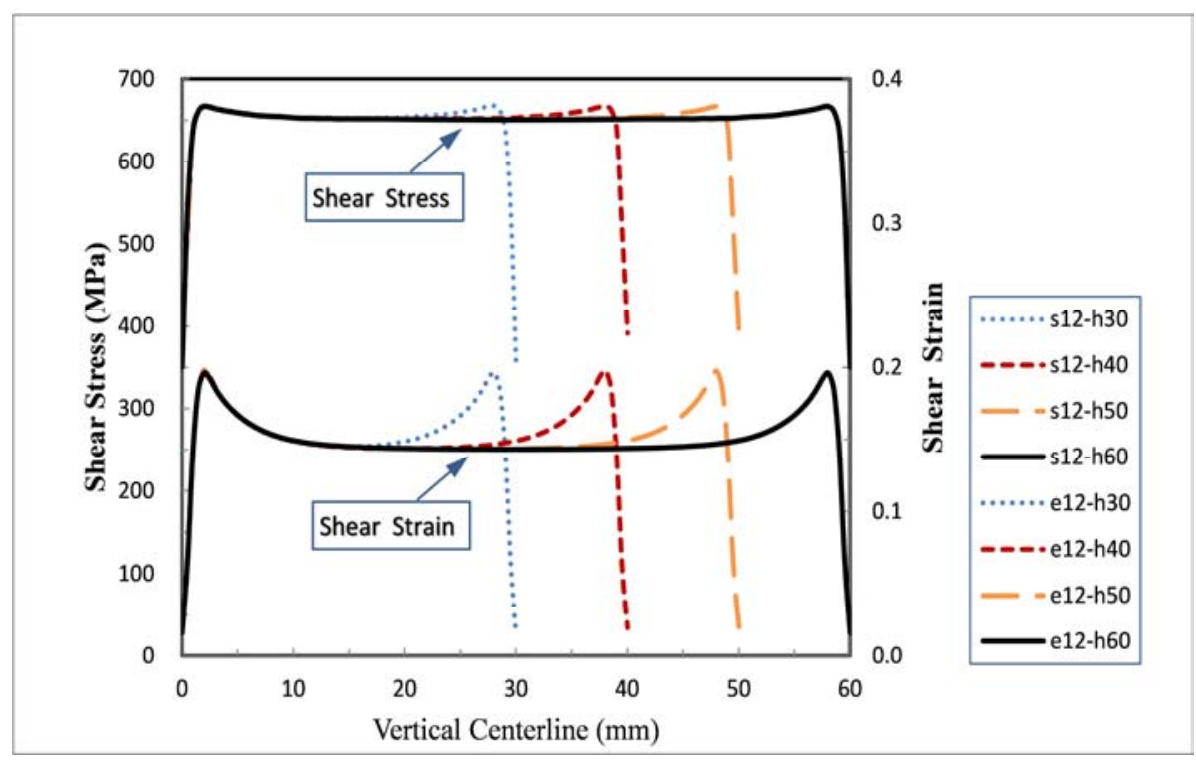

图5 剪力试件沿垂直中线之剪应力与剪应变分布图。

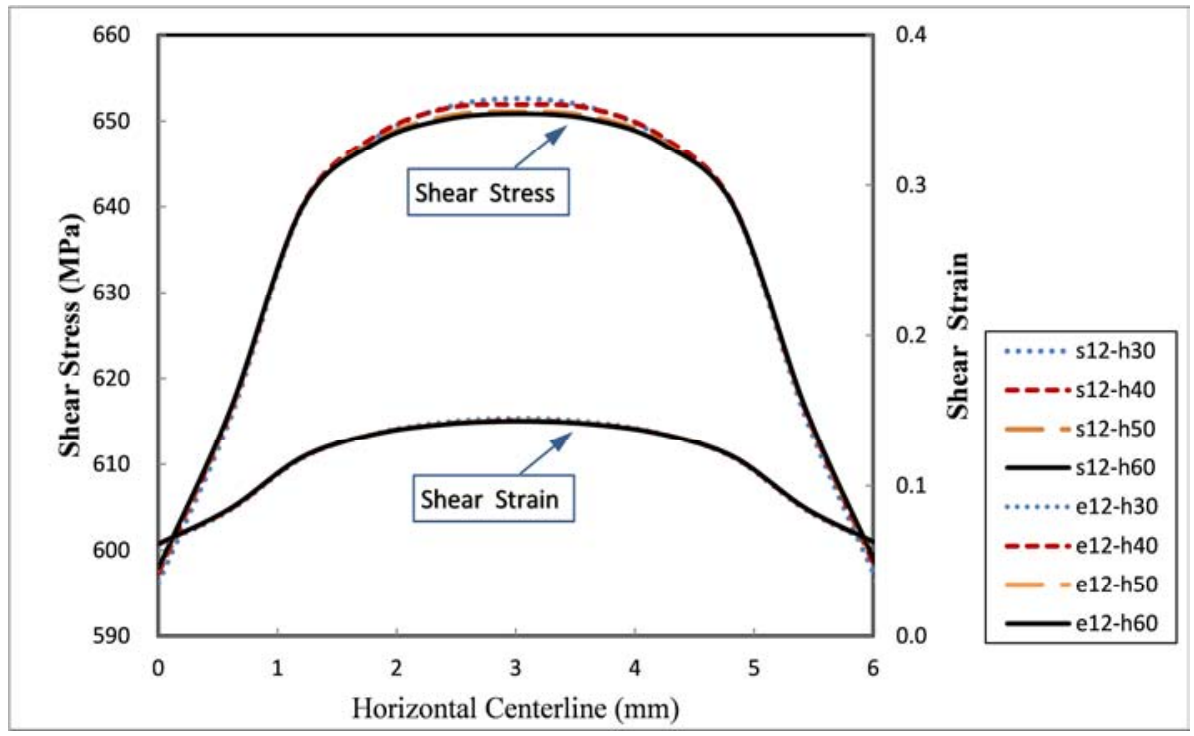

图6 剪力试件沿水平中线之剪应力与剪应变分布图。 


\section{4. 实验结果与 $\mathrm{Y}-\mathrm{U}$ 材料参数}

\section{1. 单轴向拉伸试验结果}

单轴向拉伸试验可以取得许多材料性质, 包括弹性模

数, 降伏强度, 抗拉强度, 伸长率等性质, 图 7 是四种材
料的单轴向拉伸应力应变图, 由图中可发现 JSC1180Y与 JSC980Y无明显降伏点, 而JAC590R与JSC4409P则有明显的 降伏点。

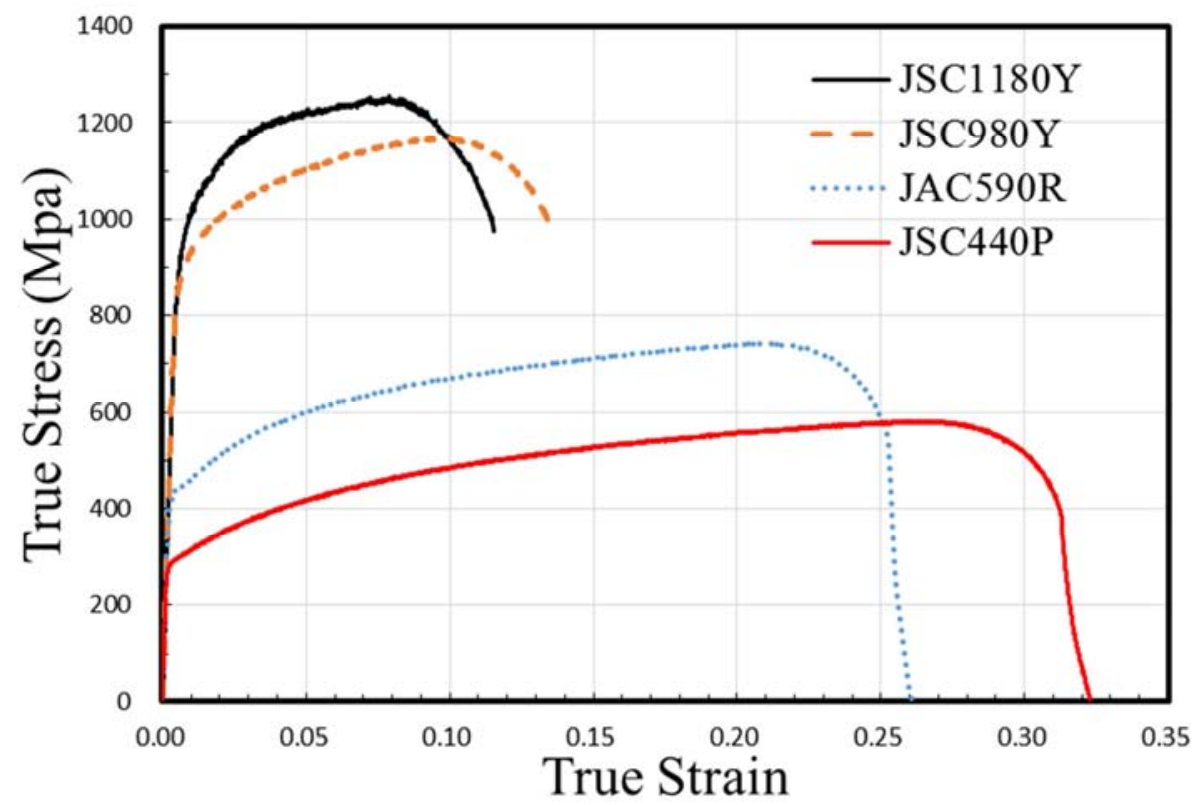

图7 四种材料的单轴向拉伸试验应力应变图。

\section{2. 往复式剪力试验结果}

往复式剪力试验必须针对每种材料进行多种不同最大剪应变量的试验, 从图8可另外观察出一个现象, 当JSC1180 完成一个循环的正向与反向变形之后, 下一循环开始有材料软化的现象, 而JSC980Y并无明显软化的现象, 但JAC590R 与JSC440P 则明显有硬化的现象。此外, JAC590R与JSC440P都与拉伸试验一样有明显的降伏点。

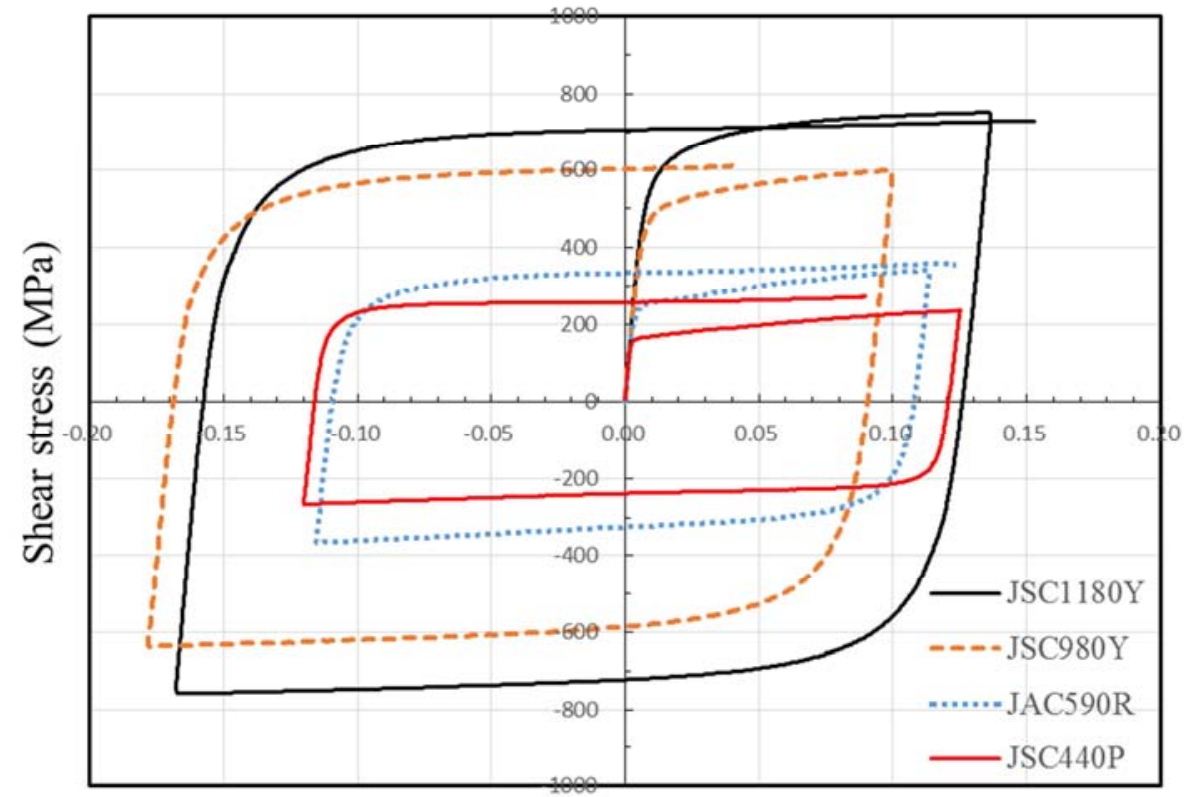

Shear Strain

图8 往复式剪力试验应力应变图。 


\section{3. 剪力试验转换为等效拉压试验}

将单轴向拉伸试验应力应变曲线, 与单向剪力试验曲 线画在同一张图上, 并且撷取单向剪力试验的应力应变, 依照 (22) 式的转换关系, 尝试不同转换系数 $F$ 之后, 可以 获得如图9的剪力试验与拉伸试验的转换关系图, 从这四 种材料的转换关系图来看, 每种材料都可以找到一个转换
系数, 使得剪力试验转成等效拉伸试验的曲线可以非常贴 近真实拉伸试验曲线, 由此可知, 剪力试验与拉伸试验确 实存有转换关系, 而且不同材料的转换系数并不相同, 必 须透过剪力试验与拉伸试验的比对才能获得, 各种材料的 转换系数如表 3 所示。

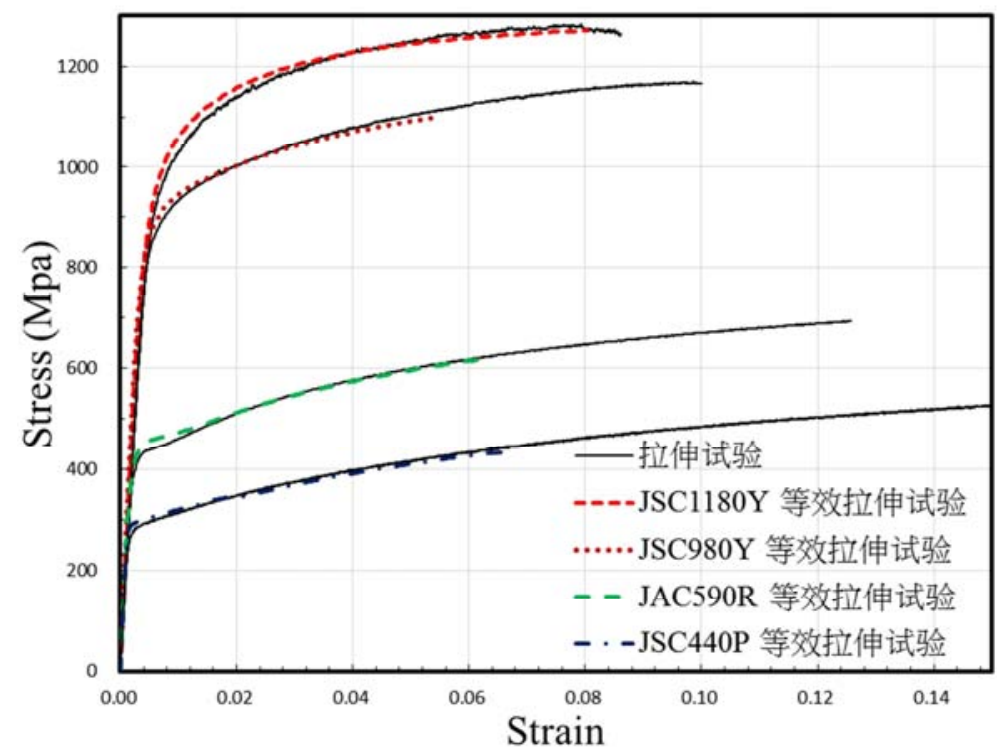

图9 剪力试验与拉伸试验的转换关系图。

表3 剪力试验转成等效拉伸试验之转换系数表。

\begin{tabular}{lllll}
\hline 材料 & JSC1180Y & JSC980Y & JAC590R & JSC 440P \\
\hline 转换系数 & 1.69 & 1.83 & 1.83 & 1.91 \\
\hline
\end{tabular}

\section{4. 等效拉压试验曲线计算 $\mathrm{Y}-\mathrm{U}$ 模型材料参数}

利用等效拉压试验曲线计算Y-U模型材料参数, 必须 将往复式剪力试验曲线转成等效拉压曲线, 并且画出真实
应力与真实塑性应变曲线图如图 10 所示, 图中的数字是回 归计算所需的测量数值。

表4是利用等效拉压试验曲线回归计算取得的四种材 料 $\mathrm{Y}-\mathrm{U}$ 模型材料参数, 详细回归计算 $\mathrm{Y}-\mathrm{U}$ 参数的方法可参考 $[12]$ ，其中 $h$ 值并非回归计算所得，必须透过实验与模拟 的结果比较才能得知, $h$ 值介于 $0 \sim 1$ 之间, 代表饱和应力的 大小, 一般而言较大的 $h$ 值代表会有较小的饱和应力, 而 较小的 $h$ 值代表会有较大的饱和应力。

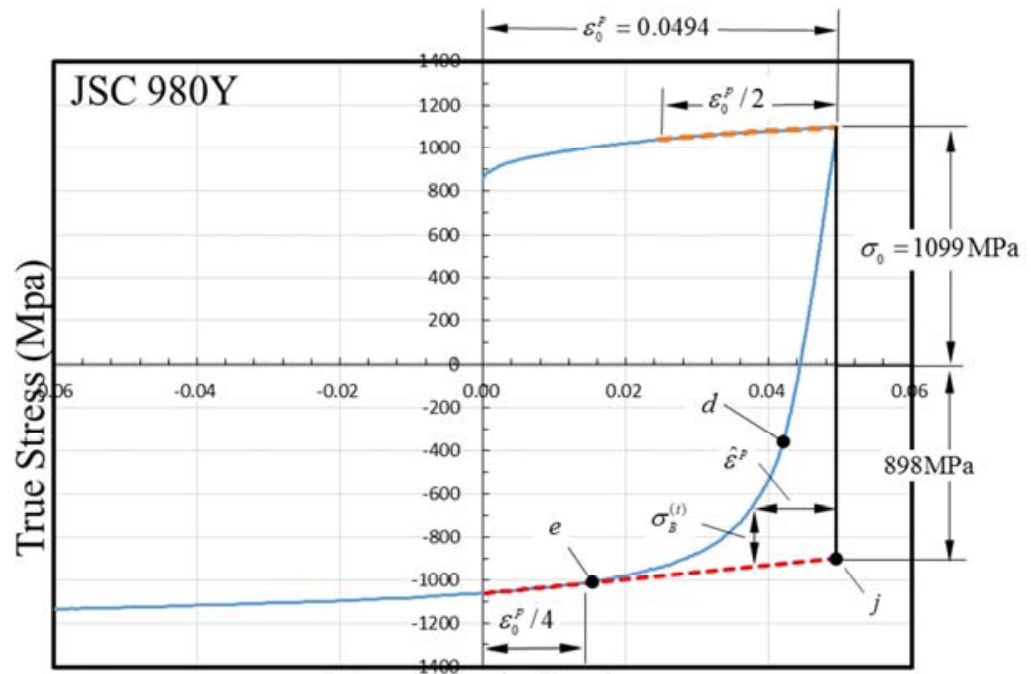

True Plastic Strain

图10 JSC980Y真实应力与真实塑性应变曲线图。 
表4 不同材料之Y-U模型参数表。

\begin{tabular}{|c|c|c|c|c|c|c|c|c|c|}
\hline 材料 & $\begin{array}{l}\mathrm{B} \\
(\mathrm{MPa})\end{array}$ & $\begin{array}{l}\text { Y } \\
\text { (Mpa) }\end{array}$ & $\mathrm{C}$ & $\mathrm{m}$ & $\begin{array}{l}\text { Rsat } \\
\text { (Mpa) }\end{array}$ & $\begin{array}{l}\mathrm{b} \\
(\mathrm{MPa})\end{array}$ & $\begin{array}{l}E a \\
(\mathrm{MPa})\end{array}$ & $\xi$ & $h$ \\
\hline JSC1180Y & 1100 & 939 & 101 & 34.2 & 106.7 & 78.5 & 167741 & 8.78 & 1 \\
\hline JSC980Y & 928 & 870 & 149 & 27. 3 & 95.3 & 135.7 & 172112 & 75.32 & 1 \\
\hline JAC590R & 443 & 421 & 173 & 16. 7 & 195.5 & 65.7 & 148276 & 8.35 & 1 \\
\hline JSC440P & 291 & 301 & 353 & 15.9 & 232.7 & -1.1 & 210679 & -32.19 & 1 \\
\hline
\end{tabular}

\section{5. 剪力试验之有限元素模拟}

利用LS-DYNA建立剪力试验模拟模型, 模拟剪力试件 变形区的中央区域, 承受均匀剪应力的作用而变形, 将其 划分成10x10网格，其边界设定如图11所示，下边界固定 $u x, u y, u z$ 自由度, 其他元素仅固定 $u y, u z$ 自由度, 因此当 上边界沿着 $x$ 方向移动时, 每个节点均能保有自由转动的 自由度, 且能模拟完整均匀剪变形的应力与应变状态。

撷取中央位置元素之剪应力与剪应变, 画出有限元素 模拟曲线与剪力试验之实验曲线比对, 四种材料之剪力试 验模拟与实验曲线比较如图12所示, 观察图中 JSC1180Y 的模拟与实验曲线, 仅有第四象限的曲线偏离实验值, JSC980Y仅有第二个巡回变形时略有偏差, 其余曲线几乎 与实验曲线完全相同, JAC590R在第四象限有较明显的误 差, JSC440P在第二、四象限瞬时包辛格区域有较明显的 误差, 一般而言, 本研究利用往复式剪力试验转换成等效 拉压试验, 以此回归获得之 $\mathrm{Y}-\mathrm{U}$ 模型参数可准确模拟循环 剪力试验之应力应变曲线。

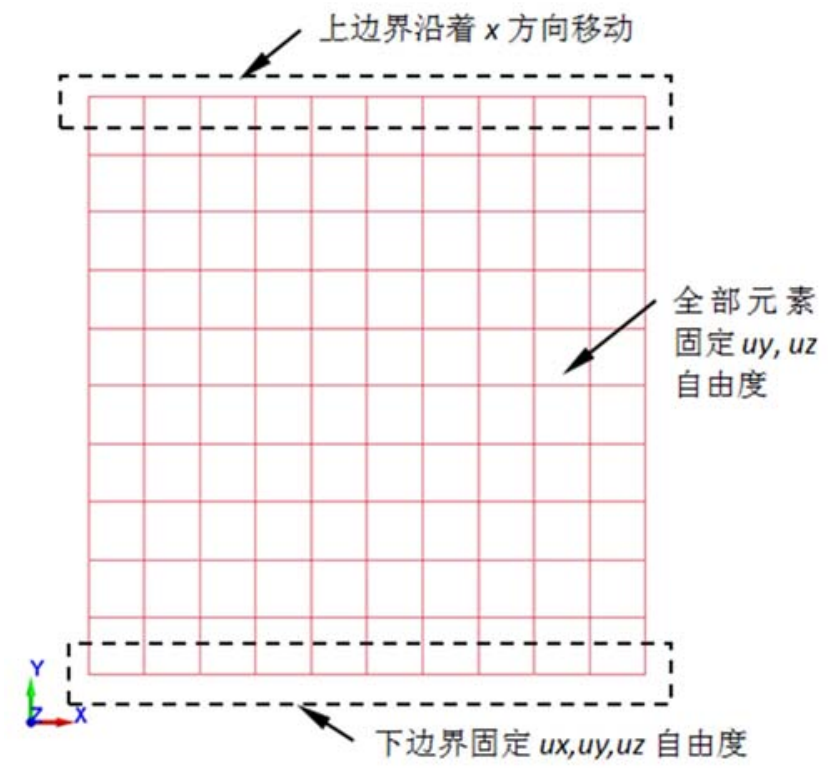

图11 LS-DYNA剪力试验模型之边界条件与网格。

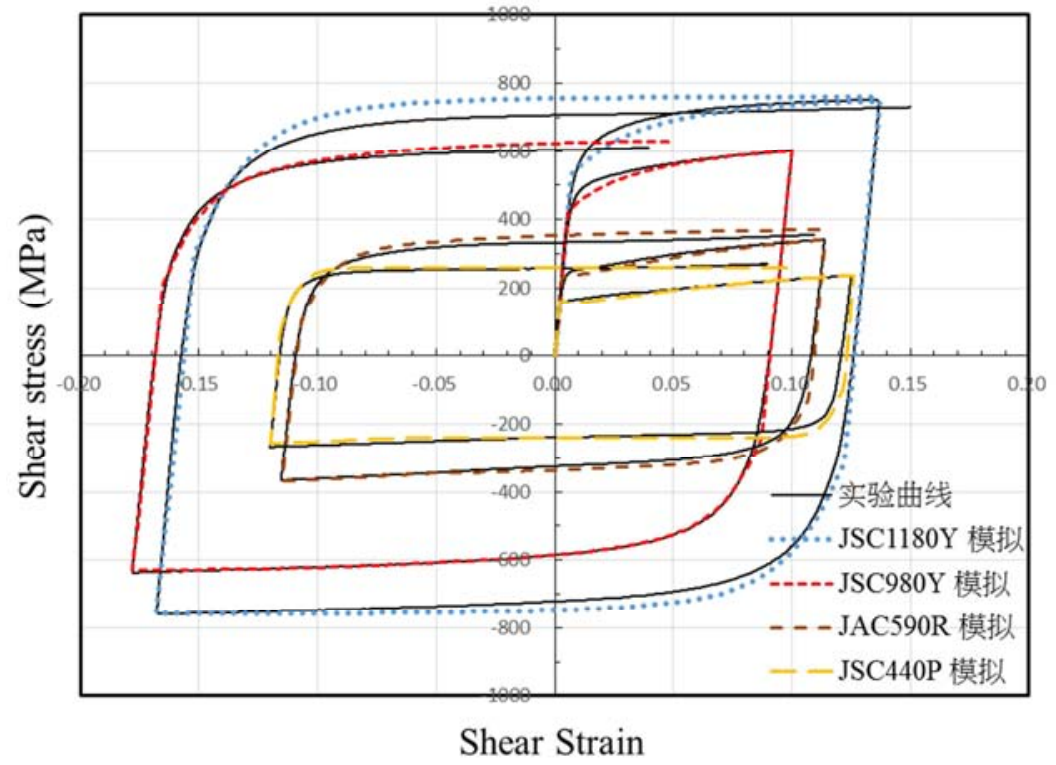

图12 剪力试验之实验与模拟曲线比较图。

\section{6. 结论}

本研究针对中钢公司所生产之JSC1180Y、JSC980Y、 JAC590R、JSC440P四种钢材进行Yoshida-Uemori材料模型 之研究, 透过拉伸试验、异向性系数量测实验、剪力试验
取得基本材料系数, 再透过单轴向拉伸试验与正向剪力试 验曲线的比对获得转换系数, 证实四种材料确实存在剪力 试验与拉伸试验的对应关系, 而其转换系数必须透过实验 曲线的比对才能取得。本研究利用往复式剪力试验转换成 的等效拉压试验曲线, 回归取得 $\mathrm{Y}-\mathrm{U}$ 材料模型参数, 最后 
以LS-DYNA有限元素模拟往复式剪力试验, 获得与实验曲 线相当一致的结果, 证实本研究所提之研究方法确实可行。

\section{致谢}

本論文为科技部计划编号 MOST 104-2622-E -253-003-CC3之计划, 由于科技部的支持, 使本计划得以 顺利进行，特此致上感谢之意。

\section{参考文献}

[1] F. Yoshida, T. Uemori and K. Fujiwara, "Elastic-plastic behavior of steel sheets under in-plane cyclic tension-compression at large strain," International Journal of Plasticity, 2002, Vol. 18, No. 5-6, pp. 633-659.

[2] F. Yoshida and T. Uemori, “A model of large-strain cyclic plasticity describing the Bauschinger effect and workhardening stagnation, " International Journal of Plasticity, 2002, Vol. 18, No. 5-6, pp. 661-686.

[3] F. Yoshida and T. Uemori, "A model of large-Strain cycle plasticity and its application to springback simulation," International Journal of Mechanical Sciences, 2003, Vol. 45, pp. 1687-1702.

[4] Ming F. Shi, Xinhai Zhu, Cedric Xia and Thomas Stoughton, "Determination of Nonlinear Isotropic/ Kinematic Hardening Constitutive Parameters for AHSS Using Tension and Compression Tests, " Proceedings of the 7th International Conference and Workshop on Numerical Simulation of 3D Sheet Metal Forming Processes, Interlaken Switzerland, 2008, pp. 137-142.

[5] Kangkang Hu, Xiongqi Peng, Jun Chen, Hongsheng Lu and Jian Zhang, "Auto-body panel springback analysis using Yoshida-Uemori model," Advanced Materials Research, 2011, Vol. 314-316, pp 815-818.
[6] A. Ghaei, D. E. Green and A. Taherizadeh, "Semiimplicit numerical integration of Yoshida-Uemori two-surface plasticity model," International Journal of Mechanical Sciences, 2010, Vol. 52, No. 4, pp. $531-540$.

[7] A. Ghaei, and D. E. Green, “Numerical implementation of Yoshida-Uemori two-surface plasticity model using a fully implicit integration scheme," Computational Materials Sciences, 2010, Vol. 48, pp. 195-205.

[8] B. Chongthairungruang, V. Uthaisangsuk, S. Suranuntchai and S. Jirathearanat, "Springback prediction in sheet metal forming of high strength steels, "Materials and design, 2013, Vol. 50, pp. 253-266.

[9] S. Tamura, S. Sumikawa, T. Uemori, H. Hamasaki and F. Yoshida, "Experimental Observation of Elasto-Plasticity Behavior of Type 5000 and 6000 Aluminum Alloy Sheets, " Materials Transactions, 2011, Vol. 52, No. 5, pp. 868-875.

[10] J. Carbonnière, S. Thuillier, F. Sabourin, M. Brunet and P. Y. Manach, "Comparison of the work hardening of metallic sheets in bending-unbending and simple shear, " International Journal of Mechanical Sciences, 2009, Vol. 51, No. 2, pp. 122-130.

[11] Chih-Yi Chang, Ming-Hsiung Ho, Ping-Chen Shen, "Yoshida-Uemori material models in cycling tension-compression and shear tests, " Proceedings of the Institution of Mechanical Engineers, Part B: Journal of Engineering Manufacture, 2014, Vol. 228(2), pp. $245-254$.

[12] 张志毅, 林义凯, 何明雄, 沈炳臣. 应用剪力试验于DP980 高强度钢之Yoshida-Uemori材料模型研究 $[\mathrm{J}]$. 桃园创新学 报，2015，第35期：pp. 49-66。

[13] Y. G. An, H. Vegter and J. Heijne, "Development of simple shear test for the measurement of work hardening, " Journal of Materials Processing Technology, 2009, Vol. 209, pp. $4248-4254$. 